\title{
Alimentação de Isopisthus parvipinnis (Teleostei: Sciaenidae) na Baía de Santos, São Paulo*
}

\author{
Lucy Satiko Hashimoto SOARES
}

Instituto Oceanográfico da Universidade de São Paulo

(Caixa Postal 9075, 01051 São Paulo, SP)

- Abstract: Stomach contents of 688 specimens of Isopisthus parvipinnis from Baía de Santos (SP) sampled between June/76 and April/77 were examined. The relative importance of different components of the diet was expressed as percentage frequency of occcurrence, percentage number, and feeding index proposed by Kawakami \& Vazzoler (1980). The most frequent and abundant items were Teleostei and Decapoda Natantia. The relative proportions of the main dietary items exhibited seasonal variations. Also, dietary changes may be linked to growth.

- Descriptors: Isopisthus parvipinnis, Sciaenidae, Stomach content, Feeding, Baía de Santos, Brazil.

- Descritores: Isopisthus parvipinnis, Sciaenidae, Conteúdo estomacal, Alimentação, Baía de Santos: SP.

\section{Introdução}

Isopisthus parvipinnis (Cuvier, 1830), vulgarmente conhecida como tortinha, constitui uma das 57 espécies de Sciaenidae conhecidas para o Atlântico Ocidental (Chao, 1978). Possui ampla distribuição geográfica, ocorrendo desde a plataforma continental da Costa Rica ao sul do Brasil, com registros até Santa Catarina (Menezes \& Figueiredo, 1980).

Existem estudos sobre a biologia e ecologia da espécie na costa brasileira, entre Cabo Frio e Torres (Soares, 1982), e sobre a composição da população e aspectos da reprodução na costa do Estado de São Paulo (Coelho et al., 1988).

Sobre a alimentação da espécie na costa brasileira não há estudo detalhado, existindo apenas uma nota preliminar (Franco, 1959) e resultados da análise de uma amostra coletada no Estuário de São Vicente, SP (Huerta-Craig, 1986). Para a costa da Guiana, LoweMcConell $(1962 ; 1966)$ a classificou como comedora de camarões pelágicos.

Este trabalho tem o intuito de contribuir para a ampliação do conhecimento da biologia da espécie no

(*) Parte da dissertação de mestrado apresentada ao Instituto Oceanográfico da Universidade de São Paulo. Em memória do Dr. Gelso Vazzoler, meu querido orientador.

Contr. $n^{2} 708$ do Inst. oceanogr. da Usp. sentido de fornecer subsídios para o delineamento das interrelaçōes tróficas no local de estudo.

\section{Material e métodos}

O estudo baseou-se na análise do conteúdo estomacal de 688 exemplares de Isopisthus parvipinnis com comprimento entre 43 e $170 \mathrm{~mm}$, coletados na Baía de Santos ( $\left.24^{\circ} 00^{\prime} \mathrm{S}, 46^{\circ} 21^{\prime} \mathrm{W}\right)$, a bordo do barco de pesquisa "Emília", usando-se rede de porta de arrasto de fundo, durante campanhas sazonais realizadas entre junho de 1976 e abril de 1977 (Tab. 1). Imediatamente após a coleta os exemplares foram fixados em formalina $10 \%$ neutralizada. De cada exemplar foi retirado o trato digestivo, conservado em álcool $70 \%$ e obtido o comprimento total e o peso total.

Para cada estômago foi anotada a presença ou ausência de alimento, a ocorrência e o número de presas de cada categoria taxonômica considerada.

Os dados foram analisados através da freqüência de ocorrência e freqüência numérica dos itens alimentares (Hynes 1950; Hyslop, 1980; Lande 1973; 1976) e através do índice alimentar (IAZ) proposto por Kawakami \& Vazzoler (1980).

Em relação à contagem dos itens alimentares foram considerados apenas organismos inteiros ou partes que permitiam individualização como, por exemplo, a presença de cefalotórax ou abdome, no caso de 
Tabela 1. Data das coletas nas quatro estações do ano, número de exemplares, amplitude de comprimento e, freqüência de estômagos com alimentos e vazios

\begin{tabular}{lcccccc}
\hline Estação do ano & N & Ax (mm) & $\begin{array}{c}\text { Com } \\
\text { N }\end{array}$ & $\begin{array}{c}\text { al imento } \\
\%\end{array}$ & Nazio & $\%$ \\
\hline $\begin{array}{l}\text { Inverno } \\
\text { (junho/1976) }\end{array}$ & 174 & $45-140$ & 140 & 80,46 & 34 & 19,54 \\
$\begin{array}{l}\text { Primavera } \\
\text { (out/nov/1976) }\end{array}$ & 239 & $43-170$ & 231 & 96,65 & 8 & 3,35 \\
$\begin{array}{l}\text { Verão } \\
\text { (março/1977) }\end{array}$ & 99 & $75-137$ & 93 & 93,94 & 6 & 6,06 \\
$\begin{array}{l}\text { Outono } \\
\text { (abril/1977) }\end{array}$ & 176 & $56-155$ & 173 & 98,3 & 3 & 1,73 \\
\hline \begin{tabular}{l} 
TotAL \\
\hline
\end{tabular} & 688 & $43-170$ & 637 & 92,58 & 51 & 7,41 \\
\hline
\end{tabular}

$\mathrm{N}=$ Número de estômagos examinados

Ax $=$ Amplitude de comprimento dos exemplares

crustáceos. Fragmentos ou estruturas, como olho, plé́podo e escamas foram computados apenas como ocorrência.

Inicialmente, a análise foi feita para exemplares de cada sexo. Como nāo se verificaram diferenças, os dados foram grupados. Procedeu-se, então, a análise:

1. por estação do ano;

2. para jovens e adultos, na primavera e outono quando ocorreram estes dois grupos bem representados;

3. para o global.

O critério adotado para separação de jovens e adultos foi o tamanho da primeira maturação gonadal (Soares, 1983). Sāo considerados indivíduos jovens, aqueles com comprimento total menor que $114 \mathrm{~mm}$.

\section{Resultados e discussão}

O espectro alimentar de Isopisthus parvipinnis, na regiāo em estudo, é constituído basicamente por três grupos zoológicos superiores: Teleostei, Crustacea e Polychaeta. O item Crustacea se constituiu de Decapoda Natantia (entre eles, Sergestidae), Brachyura, Thalassinidea, Isopoda, Gammaridea, Cumacea e Mysidacea (Tab. 2).

Com relação à composiçāo do alimento, peixes (Teleostei) e camarōes (Decapoda) são os itens principais com índice alimentar (IAi) respectivamente de 0,36 e 0,61 . Os demais componentes podem ser considerados acidentais ou ocasionais, dada sua baixa freqüência (Fig. 1).

Várias espécies de cienídeos da costa brasileira foram objeto de estudo quanto à sua alimentação. Entre elas citamos Ctenosciaena gracilicirrhus, Cynoscion jamaicensis, Cynoscion striatus, Macrodon ancylodon, Micropogonias furnieri, Paralonchurus brasiliensis e Umbrina canosai (Vazzoler, 1975; Cunningham, 1978; Amaral \& Migotto, 1980; Juras, 1984; Juras \& Yamaguti, 1985; Alvitres Castillo, 1986; Huerta-Craig, 1986).

Comparando-se o espectro alimentar e a composição do alimento de $I$. parvipinnis ao desses cienídeos, verifica-se que $I$. parvipinnis, C. jamaicensis e $M$. ancylodon são relativamente mais estenofágicas (espectro constituído de 4 a 9 itens); e constituem um grupo com hábito alimentar similar, consumindo principalmente camarōes e peixes. Entre as referidas espécies ocorrem semelhanças no aparato bucal, apresentando boca em posiçāo terminal e oblíqua, ausência de barbilhōes e presença de dentes caniniformes anteriores.

Chao \& Musick (1977) realizaram um estudo sobre correlação das estruturas alimentares com o alimento consumido pelos cienídeos, no estuário do York River, Virginia. Com base no habitat das presas-alimento, os autores classificaram as espécies em comedoras bentônicas, cujo habitat alimentar se localiza no fundo, e comedoras pelágicas, cujo habitat alimentar se localiza na coluna de água em posição superior aos dos comedores bentônicos.

Considerando-se esta classificaçāo, as características morfológicas e a composição alimentar, constituída por presas livres natantes e inclusive por camarōes 
Tabela 2. Frequêência de ocorrência e freqüência numérica dos itens alimentares encontrados nos estômagos de I. parvipinnis nas quatro estações do ano grupadas, na Bala de Santos, SP.

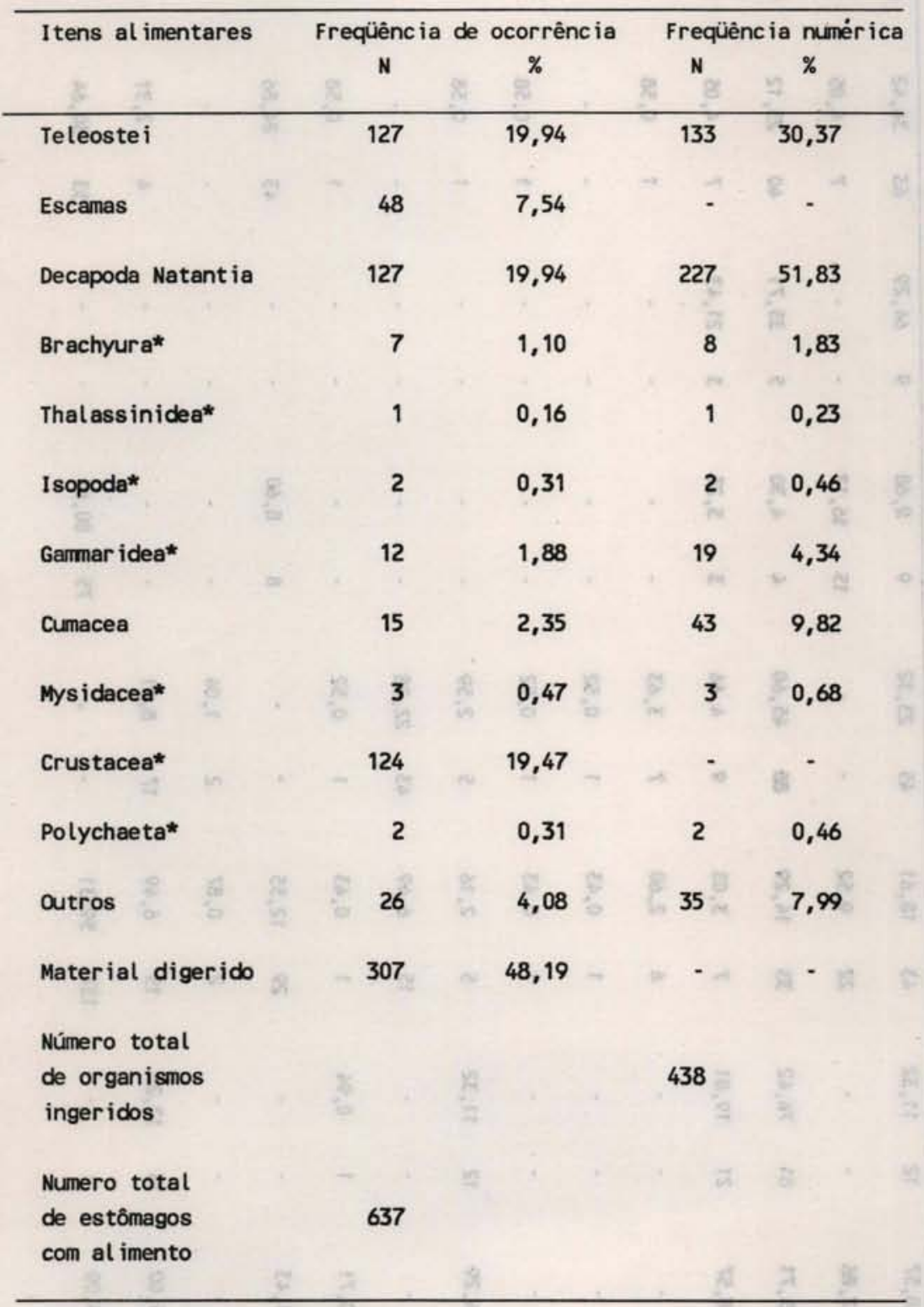

0 item "outros" compreende os itens com asterisco 


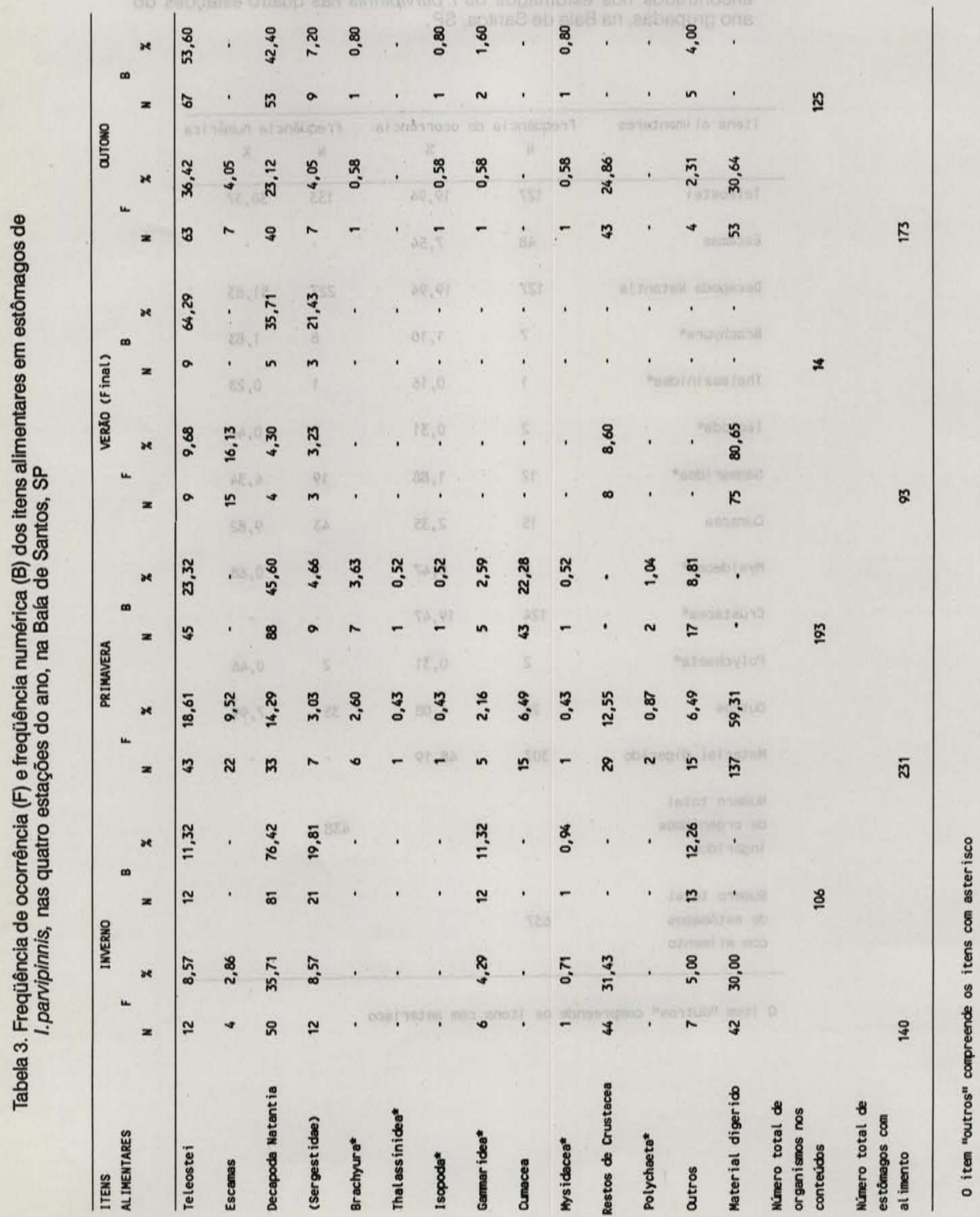




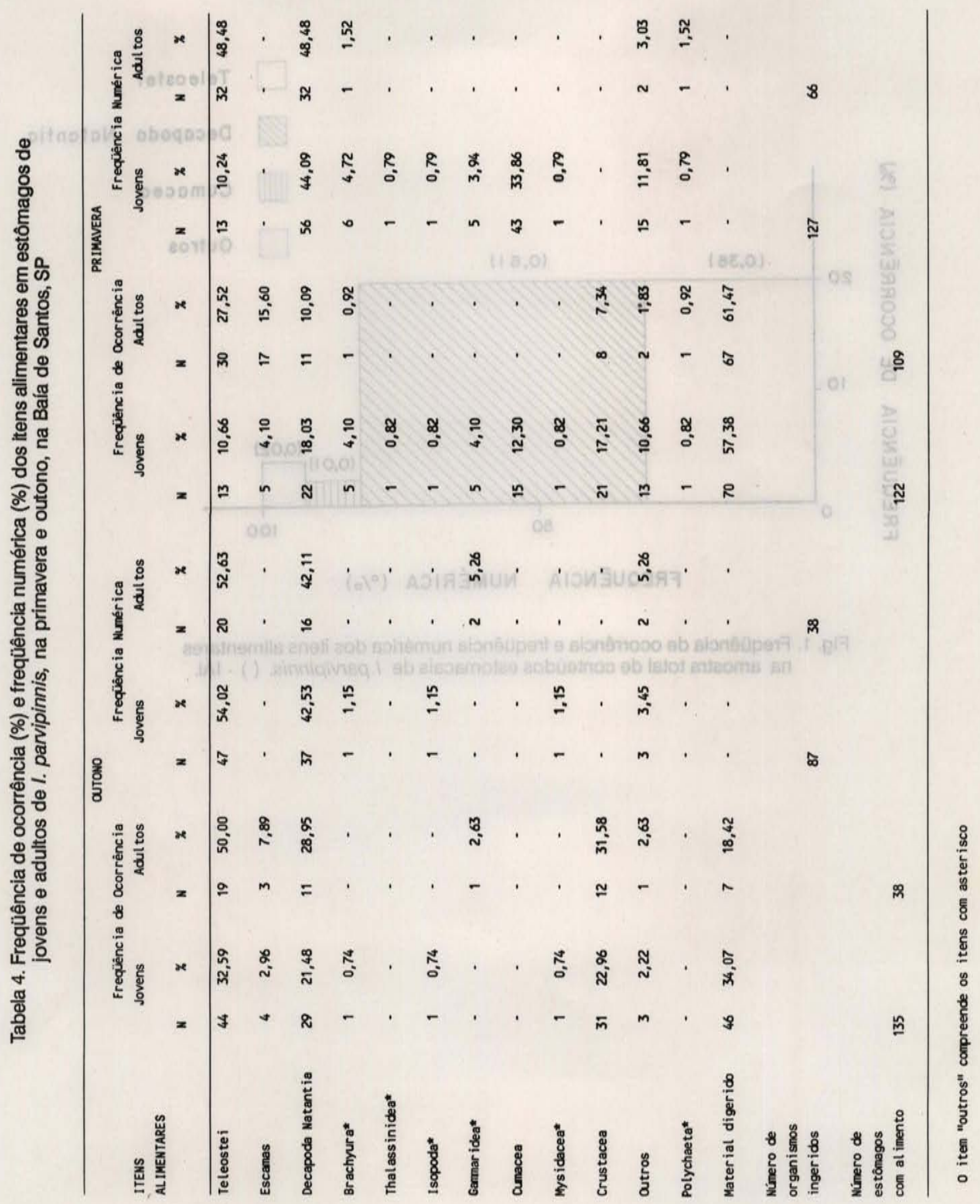




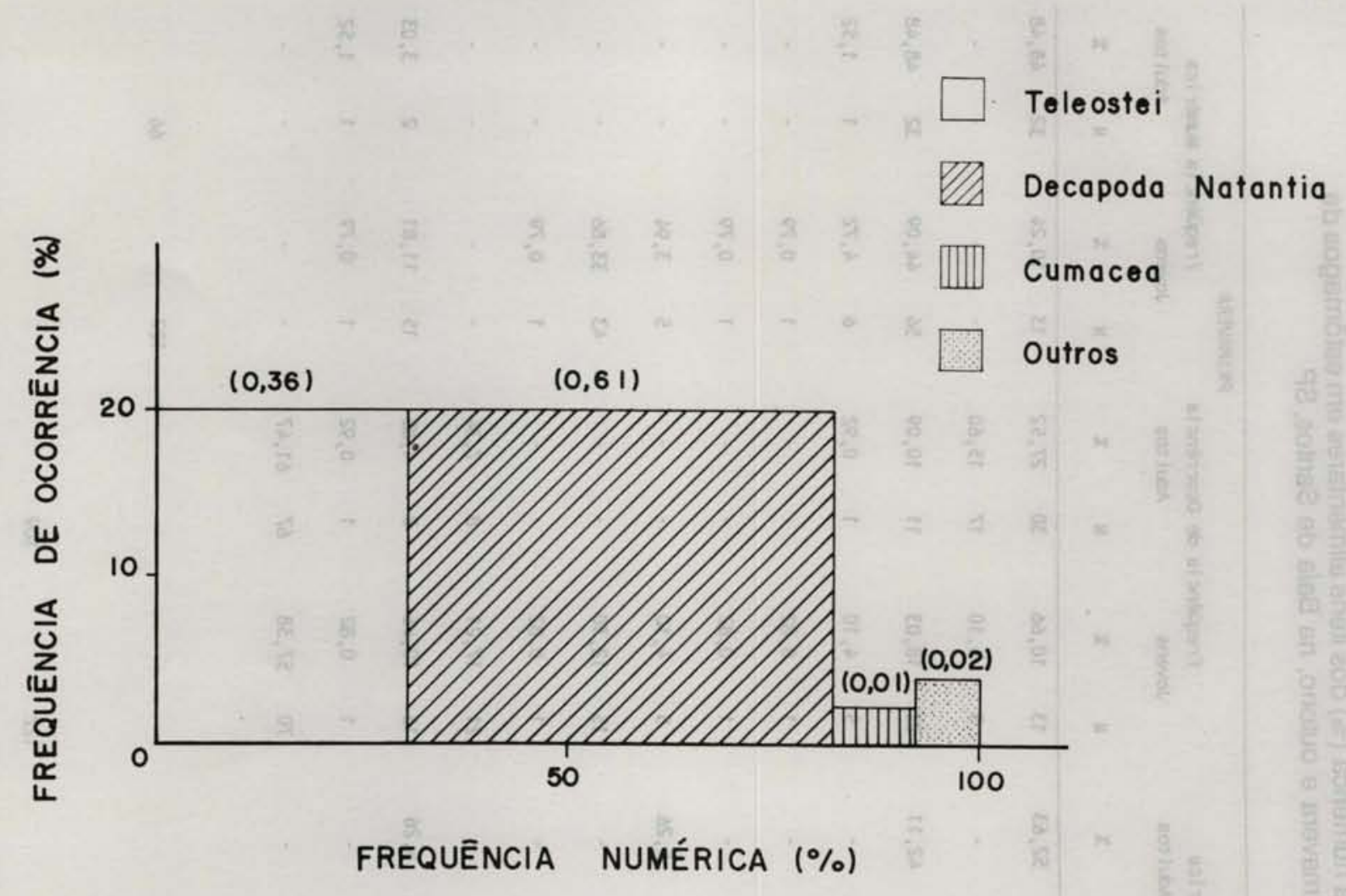

Fig. 1. Freqüência de ocorrência e freqüência numérica dos itens alimentares na amostra total de conteúdos estomacais de I.parvipinnis. () - IAi. 

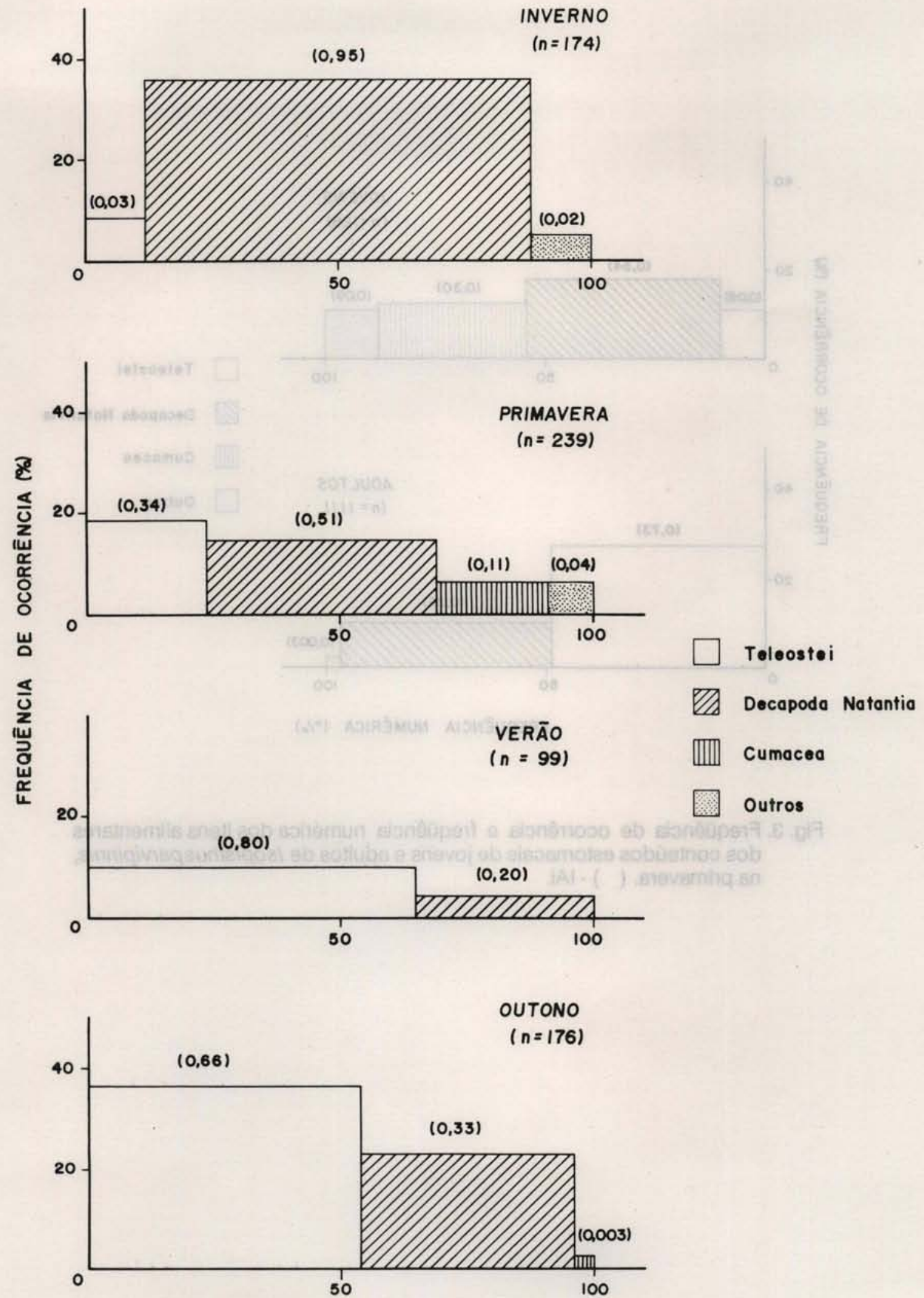

FREQUÊNCIA NUMÉRICA $(\%)$

Fig. 2. Freqüência de ocorrência e freqüência numérica dos itens alimentares em estômagos de I. parvipinnis, nas quatro estações do ano. ( ) - IAi. 


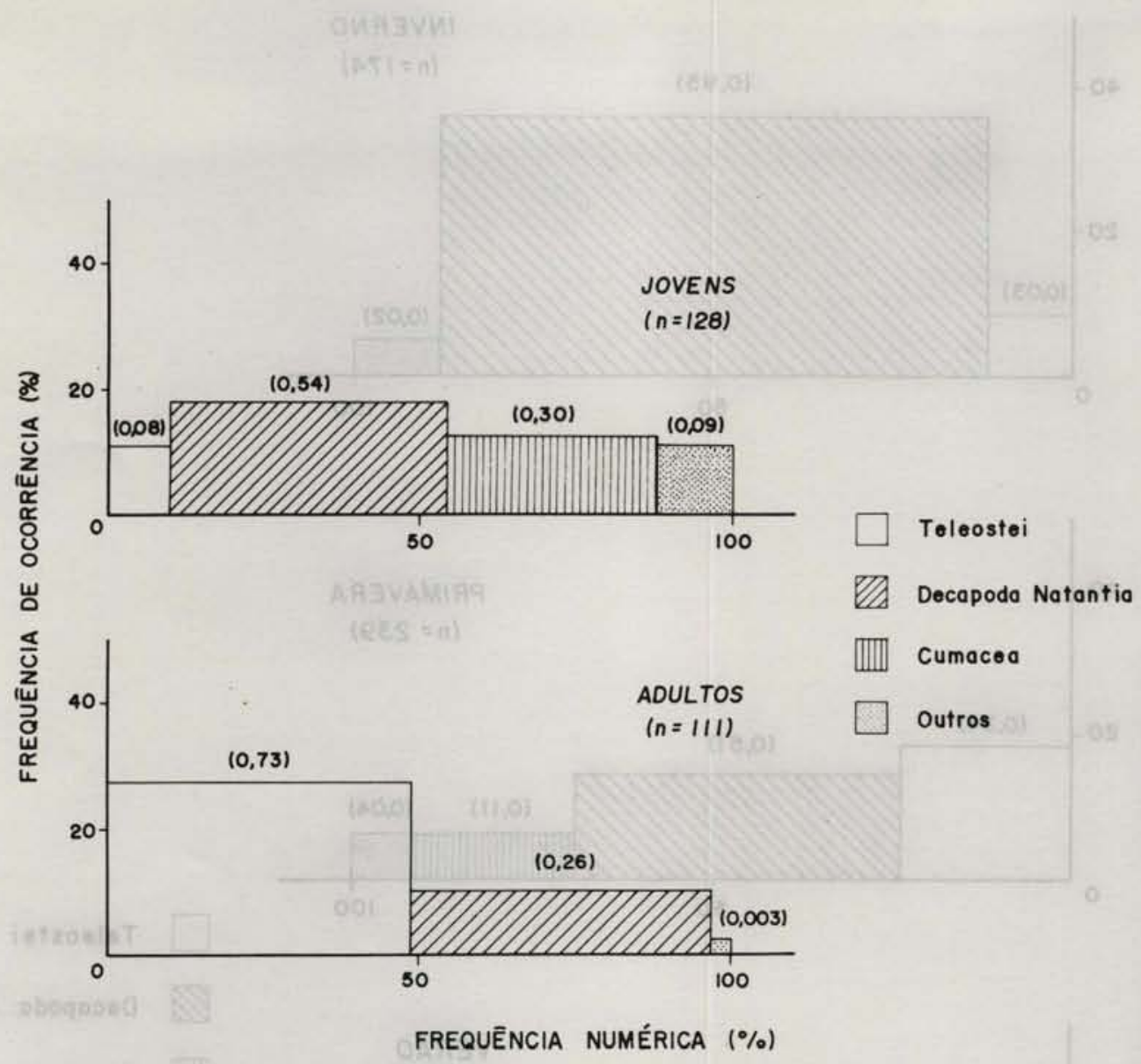

Fig. 3. Freqüência de ocorrência e freqüência numérica dos itens alimentares dos conteúdos estomacais de jovens e adultos de /sopisthusparvipinnis, na primavera. ( ) - IAi. 


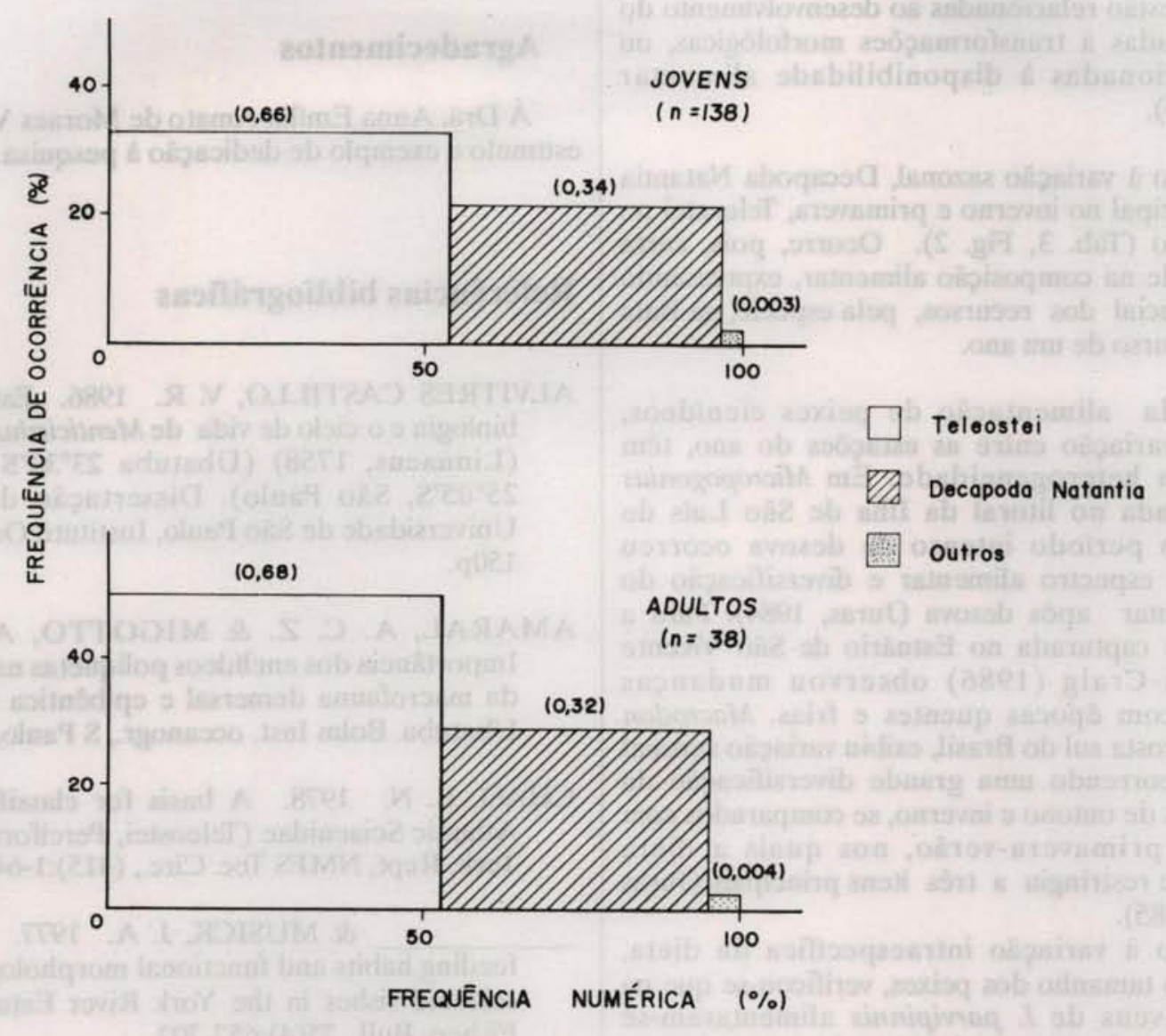

Fig. 4. Freqüência de ocorrência e freqüência numérica dos itens alimentares dos conteúdos estomacais de jovens e adultos de $I$. parvipinnis, no outono. ( ) - IAi. 
Sergestidae, $I$. parvipinnis enquadra-se no primeiro grupo, onde Chao \& Musick (op. cit.) incluiram Cynoscion regalis e Larimus breviceps.

I. parvipinnis apresentou diferenças na composição do alimento entre jovens e adultos e entre estaçōes do ano. Estas variaçōes alimentares intraespecíficas em uma mesma regiấo estão relacionadas ao desenvolvimento do peixe e associadas a transformações morfológicas, ou àquelas relacionadas à disponibilidade alimentar (Nikolsky, 1963).

Com relação à variação sazonal, Decapoda Natantia foi o item principal no inverno e primavera, Teleostei no verão e outono (Tab. 3, Fig. 2). Ocorre, pois, certa heterogeneidade na composição alimentar, expressando um uso diferencial dos recursos, pela espécie, na Baía de Santos, no curso de um ano.

Estudos da alimentação de peixes cienídeos, abordando a variaçāo entre as estaçōes do ano, têm mostrado esta heterogeneidade. Em Micropogonias furnieri capturada no litoral da Ilha de São Luís do Maranhão, no período intenso de desova ocorreu diminuição do espectro alimentar e diversificação do regime alimentar após desova (Juras, 1984). Para a mesma espécie capturada no Estuário de São Vicente (SP), Huerta-Craig (1986) observou mudanças relacionadas com épocas quentes e frias. Macrodon ancylodon, da costa sul do Brasil, exibiu variação sazonal significante, ocorrendo uma grande diversificação da dieta nos meses de outono e inverno, se comparados com os meses de primavera-verão, nos quais a dieta praticamente se restringiu a três itens principais (Juras \& Yamaguti, 1985).

Em relação à variação intraespecífica da dieta, considerando o tamanho dos peixes, verificou-se que na primavera, jovens de $I$. parvipinnis alimentaram-se principalmenmte de Decapoda Natantia e Cumacea e os adultos, de Teleostei (Tab. 4, Fig. 3). No outono, jovens e adultos consumiram principalmente Teleostei (Tab. 4, Fig. 4). Mudanças na composiçâo do alimento relacionadas com o crescimento são observadas em diversas espécies de peixes. No caso do cienídeo Macrodon ancylodon, os imaturos alimentam-se principalmente de camarōes Artemia longinaris e os maduros, de peixes (Juras \& Yamaguti, 1985). Em Micropogonias fumieri, de modo geral, os imaturos se alimentam de poliqueta, e os maduros, de peixes (Juras, 1984).

Do exposto pode-se depreender que, embora a espécie em questão exiba variaçōes intraespecíficas, seu habitat trófico, tanto do estrato jovem como do adulto, se localiza no corpo de água.

\section{Resumo}

Conteúdos estomacais de 688 exemplares de Isopisthus parvipinnis coletados na Baía de Santos (SP) $\left(24^{\circ} 00^{\prime} \mathrm{S}, 46^{\circ} 21^{\prime} \mathrm{W}\right)$ entre junho/76 e abril/77 foram examinados. A importância relativa dos componentes da dieta foi medida pela freqüência de ocorrência, freqüência numérica e pelo uso do índice alimentar proposto por Kawakami \& Vazzoler (1980). Verificou-se que a espécie se alimenta principalmente de Teleostei e Decapoda Natantia, apresentando hábito alimentar pelágico. Variaçōes intraespecíficas foram observadas tanto entre as estaçōes do ano como entre os estratos jovem e adulto.

\section{Agradecimentos}

Á Dra. Anna Emília Amato de Moraes Vazzoler pelo estímulo e exemplo de dedicação à pesquisa.

\section{Referências bibliográficas}

ALVITRES CASTILLO, V. R. 1986. Estudo sobre a biologia e o ciclo de vida de Menticinhus americanus (Linnaeus, 1758) (Ubatuba 2330'S - Cananéia $25^{\circ} 05^{\prime}$, São Paulo). Dissertaçāo de mestrado. Universidade de São Paulo, Instituto Oceanográfico. 150p.

AMARAL, A. C. Z. \& MIGOTTO, A. E. 1980. Importância dos anelídeos poliquetas na alimentação da macrofauna demersal e epibêntica da regiāo de Ubatuba. Bolm Inst. oceanogr., S Paulo, 29(2):31-35.

CHAO, L. N. 1978. A basis for classifying western Atlantic Sciaenidae (Teleostei, Perciformes). NOAA Tech. Rept, NMFS Tec. Circ., (415):1-64.

\& MUSICK, J. A. 1977. Life history, feeding habits and functional morphology of juvenile sciaenid fishes in the York River Estuary, Virginia. Fishery Bull., 75(4):657-702.

COELHO, J. A. P.; GRAÇA LOPES, R. da; RODRIGUES, E. S. \& PUZZI, A. 1988. Aspectos biológicos e pesqueiros do Sciaenidae Isopisthus parvipinnis (Cuvier, 1830) Teleostei, Perciformes, Sciaenidae presente no rejeitado da pesca artesanal dirigida ao camarão sete-barbas (São Paulo, Brasil). Bolm Inst. Pesca, S Paulo, 15(1):99-108.

CUNNINGHAM, P. T. M. 1978. Bionomia e ciclo de vida de Ctenosciaena gracilicirrhus (Metzelaar, 1919) da plataforma continental brasileira entre as latitudes de $22^{\circ} 10^{\prime}$ e $30^{\circ} \mathrm{S}$. Dissertaçāo de mestrado. Universidade de São Paulo, Instituto Oceanográfico. 106p.

FRANCO, G. T. 1959. Nota preliminar sobre alimentação de alguns peixes comerciais brasileiros. Anais Acad. bras. Ciênc., 31(4):589-593.

HUERTA-CRAIG, I. D. 1986. Estudo sobre alimentação de espécies de peixes das famílias Ariidae, Carangidae, Gerreidae, Sciaenidae, Trichiuridae, Bothidae e Soleidae no estuário de São Vicente, SP. Dissertaçāo de mestrado. Universidade de São Paulo, Instituto Oceanográfico. 224p. 
HYNES, H. B. N. 1950. The food of freshwater sticklebacks (Gasterosteus aculeatus and Pygosteus pungitius), with a review of methods used in studies of the food of fishes. J. Anim. Ecol., 19(1):36-58.

HYSLOP, E. J. 1980. Stomach contents analysis - a review of methods and their application. J. Fish Biol., $17(4): 411-429$.

JURAS, A. A. 1984. Estudo sobre reprodução, regime alimentar e crescimento de Micropogonias furnieri (Desmarest, 1823) (Teleostei-Sciaenidae) capturada no litoral da Ilha de São Luis do Maranhão, Brasil. Tese de doutorado. Universidade de São Paulo, Instituto Oceanográfico. 205p.

\& YAMAGUTI, N. 1985. Food and feeding habits of king weakfish, Macrodon ancylodon (Block \& Schneider, 1801) caught in the southern coast of Brazil. Bolm Inst. oceanogr., S Paulo, $33(2): 149-157$.

KAWAKAMI, E. \& VAZZOLER, G. 1980. Método gráfico e estimativa do índice alimentar aplicado no estudo da alimentação de peixes. Bolm Inst. oceanogr., S Paulo, 29(2):205-207.

LANDE, R. 1973. Food and feeding habits of plaice (Pleuronectes platessa L.) in Borgenfjorden, North-Trondelag, Norway. Norw. J. Zool., 21(2):91-100.

1976. Food and feeding habits of the dab (Limanda limanda L.) in Borgenfjorden, North-Trondelag, Norway. Norw. J. Zool., 24(3):225-230.
LOWE-McCONELL, R. H. 1962. The fishes of the British Guiana continental shelf of Atlantic coast of South America, with notes on their natural history. J. Linn. Soc., Zool., 44(301):669-700.

1966. The sciaenid fish of British Guiana. Bull. mar. Sci. Gulf Caribb., 16:20-57.

MENEZES, N. A. \& FIGUEIREDO, J. L. 1980. Manual de peixes marinhos do Sudeste do Brasil. IV. Teleostei (3). São Paulo, Museu de Zoologia da Universidade de São Paulo. 96p.

NIKOLSKY, G. V. 1963. The ecology of fishes. London, Academic Press. 352p.

SOARES, L. S. H. 1983. Aspectos da biologia e ecologia de Isopisthus parvipinnis (Cuvier, 1830) (Perciformes: Sciaenidae) entre Cabo Frio e Torres, Brasil. Dissertação de mestrado. Universidade de São Paulo, Instituto Oceanográfico. 123p.

VAZZOLER, G. 1975. Distribuiçâo da fauna de peixes demersais e ecologia dos Sciaenidae da plataforma continental brasileira, entre as latitudes $29^{\circ} 11^{\prime} \mathrm{S}$ (Torres) e $33^{\circ} 41^{\prime} \mathrm{S}$ (Chuí). Bolm Inst. oceanogr., S Paulo, 24(único):85-169.

(Recebido em 14-10-88; aceito em 16-10-89) 